\title{
Computerized Tomography of the Acute Left Upper Quadrant Pain
}

Authors

1. Temel Tirkes, M.D. ${ }^{(1,2)}$

Associate Professor of Radiology

2. Zachary Ballenger, M.D. ${ }^{(1)}$

3. Scott D. Steenburg, M.D. ${ }^{(1)}$

Associate Professor of Radiology

4. Daniel J. Altman, M.D. ${ }^{(1)}$

5. Kumaresan Sandrasegaran, M.D. ${ }^{(1)}$

Associate Professor of Radiology

(1) Indiana University School of Medicine

Department of Radiology and Imaging Sciences

(2) Corresponding Author:

Temel Tirkes, M.D.

Associate Professor of Radiology

Indiana University School of Medicine

Department of Radiology and Imaging Sciences

550 N. University Blvd, UH 0663

Indianapolis, IN 46202

Tel: (317) 944-8832

Fax: (317) 944-1848

This is the author's manuscript of the article published in final edited form as:

Tirkes, T., Ballenger, Z., Steenburg, S. D., Altman, D. J., \& Sandrasegaran, K. (2016). Computerized tomography of the acute left upper quadrant pain. Emergency Radiology, 23(4), 353-356.

https://doi.org/10.1007/s10140-016-1410-5 


\section{ABSTRACT}

\section{OBJECTIVE}

The purpose of this study was to evaluate the clinical utility of computerized tomography (CT) of the abdomen in the emergent setting of left upper quadrant pain.

\section{METHODS}

One hundred patients (average age: 45, range: 19-93 years, female: 57\%, male: 43\%) who presented to the emergency department (ED) and underwent CT scanning of abdomen with the given indication of left upper quadrant pain were included in this study. The results from CT examinations were compared to final diagnoses determined by either ED physician or clinician on a follow-up visit.

\section{RESULTS}

Sensitivity of CT was 69\% (95\%CI: 52\%-83\%) for 39 patients who eventually were diagnosed with an acute abdominal abnormality. Twenty-seven patients had an acute abnormal finding on abdominal CT that represented the cause of the patient's pain (positive predictive value of 100\%, 95\%CI: 87\%-100\%). Of the remaining 73 patients with negative CT report, 12 were diagnosed clinically (either in the ED or on follow-up visit to specialist) with a pathology that was undetectable on the CT imaging (negative predictive value of $83 \%, 95 \% \mathrm{CI}$ : $73 \%-91 \%$ ). None of the remaining 61 patients with negative CT were found to have pathology by clinical evaluation (Specificity of 100\%, 95\%CI: 94\%-100\%).

\section{CONCLUSION}


CT is a useful examination for patients with acute left upper quadrant pain in the emergency department setting with moderate sensitivity and excellent specificity.

Keywords: Left upper quadrant; Acute abdomen; Computerized Tomography; Emergency Department 


\section{INTRODUCTION}

Abdominal pain is the most common reason for a visit to the emergency department, comprising $7 \%$ of all complaints in the emergency departments (ED) and accounting for approximately 8 million ED visits annually [1]. Causes of abdominal pain vary widely, ranging from transient, non-life-threatening conditions such as gastroenteritis and urinary tract infections, to severe, lifethreating conditions such as abdominal aortic aneurysm rupture and bowel ischemia. The location of the abdominal pain can often help to limit the differential diagnosis, however with left upper quadrant pain the potential causes vary widely, including cardiac, gastric, pancreatic, renal and vascular etiologies [1]. Current clinical practice guidelines offer mixed recommendations with regard to imaging patients with acute left upper quadrant pain, with some authors recommending $\mathrm{CT}$ of the abdomen [2] however there have been no formal studies in the radiology literature to support this practice. Some authors stating clearly that there is no specific recommended radiologic test to evaluate acute left upper quadrant pain [3]. Given that CT use in emergency departments in the United States is historically high, and continues to increase at a higher rate than in other settings [4], scientific inquiry into the utility of this practice is warranted. Having very high specificity in the emergency setting may result in decreased health care costs by avoiding additional diagnostic tests. Thus, the purpose of this study was to determine the utility of abdominal $\mathrm{CT}$ in the setting of acute left upper quadrant abdominal pain in a setting of an urban emergency department.

\section{MATERIALS AND METHODS}

The Institutional Review Board (IRB) approved this retrospective study and the requirement for informed consent for the patient data review was waived. A HIPAA compliant retrospective 
analysis was performed on 100 consecutive patients who presented to the emergency department affiliated with our academic institution over 18 months period from June 2014 to November 2015 with the chief complaint of left upper quadrant pain.

All scans were performed on a 64 slice MDCT scanner from the domes of the diaphragm to the ischial tuberocities, with images reconstructed at $3 \mathrm{~mm}$ slice thickness in the axial, sagittal and coronal planes. Sixty-eight CT examinations were performed as a single-phase intravenous contrast only; $100 \mathrm{ml}$ of Iopamidol 370 (Bracco Diagnostics Inc., Monroe Township, NJ 08831) while remaining $32 \mathrm{CTs}$ were performed without intravenous contrast. Oral contrast was not administered in many of these examinations per routine emergency CT protocol; based on the studies have shown that administration of oral contrast in the emergency setting results in significant delay to imaging acquisition without adding much diagnostic value [5]

Patients were identified by interactive data-mining software, which was used to search CT scans with an indication of "left upper quadrant pain." Patients who were less than 18 years of age, pregnant, or who went directly to surgery were excluded from analysis. All CT examinations were interpreted by board certified radiologists with experience of 8 to 19 years working in the emergency department. The primary diagnosis provided in impression section of the report considered being the final radiologic diagnosis. The electronic medical records were searched to determine the final diagnosis, using clinical exam, follow up visits, surgical findings and follow up imaging as the aggregate reference standard. The radiologic diagnosis for each patient was compared to the final clinical diagnosis and statistical analysis was performed to find out sensitivity, specificity, positive predictive value and negative predictive value of CT in diagnosis of left upper quadrant pain. 


\section{RESULTS}

The study consisted of 100 patients ( $57 \%$ female and $43 \%$ male) with an average age of 45 years (range 19-93 years). 39 patients were diagnosed with an etiology either at the time of discharge from the ED or during the follow-up visits to a specialist. Of these 39 patients, CT result was positive in 27 of them (sensitivity of 69\%, 95\%CI: 52\%-83\%) and all of these results matched with the final clinical diagnosis recorded in the patients' medical records (positive predictive value of $100 \%, 95 \%$ CI: $87 \%-100 \%)$. Renal stone disease, pancreatitis and diverticulitis were the three most common findings, accounting for $41 \%$ of the diagnoses made by CT. A list of the 27 true positive radiologic diagnoses made on these examinations is found in Table 1.

Of the remaining 73 examinations with negative CT reports, 61 patients were discharged from the emergency department with no clinical diagnosis for the left upper quadrant pain (negative predictive value $83 \%, 95 \%$ CI: 73\%-91\%). However, the remaining 12 patients were diagnosed clinically with pathology. Gastritis, pancreatitis and urinary tract infection were among the most common underlying etiologies of left upper quadrant pain that were not detected on CT examination. A list of false negative results is in Table 2. Follow-up examinations were performed in 42 patients. These included non-emergent radiologic examinations (e.g. MR cholangiopancreatography, lumbar MR, x-ray or ultrasound) or examinations in specialists' office (e.g. upper GI endoscopy, ureteroscopy).

\section{DISCUSSION}

Acute abdominal pain is the most common chief complaint for which patients seek care in emergency departments in the United States [1]. History and physical examination may be helpful in limiting the differential diagnosis for the emergency physician, however a definite 
diagnosis may remain elusive and thus imaging and laboratory testing are commonly ordered for further evaluation of the patient's pain [6]. This is particularly true for left upper quadrant pain, which is caused by a wide variety of clinical conditions [2]. Anatomically, the left upper quadrant contains the following structures; stomach, spleen, left liver lobe, jejunum, proximal ileum, pancreas (body and tail), left kidney, left adrenal gland, and colon (left half of transverse colon, splenic flexure and proximal descending colon) [7]. Clinical guidelines often recommend imaging of the abdomen with CT for the acute presentation of LUQ pain, given the relatively broad differential diagnosis for left upper quadrant abdominal pain, some of which are potentially life-threatening [6]. While renal and adrenal causes of abdominal pain more classically localize to the flanks, acute disease in these organs results in referred pain to the upper quadrants often enough to merit inclusion in the differential diagnosis [8]. The American College of Radiology's Appropriateness Criteria are available for “Acute (non-localized) Abdominal Pain and Fever or Suspected Abdominal Abscess" [9], "Left Lower Quadrant PainSuspected Diverticulitis"[10], "Right Upper Quadrant Pain" [11], and "Right Lower Quadrant Pain, Suspected Appendicitis" [12] but there is no criteria that specifically address left upper quadrant abdominal pain. Therefore radiologists are well positioned to lead the way in investigating the utility of imaging patients with acute left upper quadrant pain with $\mathrm{CT}$, and to either support this practice or recommend against it depending on what is most effective for the patients.

In this study, we reviewed $100 \mathrm{CT}$ examinations performed over 18 months for evaluation of left upper quadrant pain at emergency department of a major academic institution. The results of these imaging examinations, referred to in this document as the radiologic diagnoses were compared to the patients' clinical diagnoses found in the discharge materials of the patients' 
electronic medical records. These results were analyzed to evaluate the utility of using CT of the abdomen and pelvis in the setting of acute left upper quadrant pain.

The results of this investigation revealed sensitivity for the CT abdomen and pelvis to be $69 \%$, which is moderately good in the setting of acute left upper quadrant pain. However, specificity was $100 \%$, indicating that if a specific diagnosis is made on CT examination, it is highly likely to represent the underlying pathology of the left upper quadrant pain. Some of the more common positive radiologic diagnoses, renal stone disease and diverticulitis, are typically associated with other locations of abdominal pain (left lower quadrant and flank, respectively), adding support to the assertion that localization of abdominal pain in an attempt to refine a differential diagnoses is often inaccurate, and imaging with $\mathrm{CT}$ is appropriate. CT examination had 12 false negative cases. Review of these results from Table 2 reveals that more than half of these false negative results involved esophagitis, gastritis or gastric anastomotic ulcer; diagnoses for which CT is often not the primary investigative modality [13]. Pancreatitis and urinary tract infection were other clinical diagnoses that were classified as false negative results in this examination; however these are much more likely to be detected in routine laboratory tests in acute setting of abdominal pain. We find that imaging patients who present with acute left upper quadrant pain using $\mathrm{CT}$ of the abdomen and pelvis is an appropriate practice as well as practical.

Limitations of this study include that the study was retrospective in design. Study included adult patients only. Both contrast enhanced and unenhanced CT scans of the abdomen and pelvis were included in the study. Decision to perform the non-enhanced CT was primarily dependent on the clinicians' high suspicion of renal stone as the etiology, the presence of allergy to iodinated contrast media and renal impairment as a contraindication to CT contrast media. 


\section{CONCLUSION}

Abdominal CT had 100\% specificity and 69\% sensitivity for finding the etiology of acute left upper quadrant pain. The underlying etiologies of left upper quadrant pain are quite variable. The most common etiologies that were correctly diagnosed with CT included renal stones, pancreatitis, diverticulitis, hernia, pneumonia and rib fractures. The most common etiologies that were not detected on CT were gastritis, pancreatitis, urinary tract infection and esophagitis. 


\section{Tables}

Table 1. Radiologic diagnoses correctly made on CT examination in patients with left upper quadrant pain.

Diagnosis

Incidence $(\%)$

Nephrolithiasis/Urolithiasis

5

Pancreatitis

4

Diverticulitis

3

Rib fracture

3

Abdominal wall hernia

2

Gastritis/Enteritis

2

Pneumonia $+/$ - pleural effusion

2

Duodenitis

1

Bowel perforation

1

Epiploic appendagitis

1

Small bowel obstruction

1

Ruptured splenic cyst

1

Splenic laceration

1 
Table 2. Etiologies of left upper quadrant pain that were not detected by CT of the abdomen.

Final Clinical Diagnosis

Gastritis/Gastric ulcer

Urinary tract infection

Pancreatitis

Esophagitis

Bariatric surgery with anastomotic ulcer

Pulmonary infarct

\section{Incidence (\%)}

\section{5}

2

2

1

1

1

\section{Financial Disclosure}

The authors declare that they have no conflict of interest. 


\section{References}

1. Macaluso CR, McNamara RM (2012) Evaluation and management of acute abdominal pain in the emergency department. International journal of general medicine 5:789-797. doi:10.2147/IJGM.S25936

2. Cartwright SL, Knudson MP (2008) Evaluation of acute abdominal pain in adults. American family physician 77 (7):971-978

3. Panebianco NL, Jahnes K, Mills AM (2011) Imaging and laboratory testing in acute abdominal pain. Emerg Med Clin North Am 29 (2):175-193, vii. doi:10.1016/j.emc.2011.01.010 4. Larson DB, Johnson LW, Schnell BM, Salisbury SR, Forman HP (2011) National trends in CT use in the emergency department: 1995-2007. Radiology 258 (1):164-173. doi:10.1148/radiol.10100640

5. Lee SY, Coughlin B, Wolfe JM, Polino J, Blank FS, Smithline HA (2006) Prospective comparison of helical CT of the abdomen and pelvis without and with oral contrast in assessing acute abdominal pain in adult Emergency Department patients. Emerg Radiol 12 (4):150-157. doi:10.1007/s10140-006-0474-Z

6. Gerhardt RT, Nelson BK, Keenan S, Kernan L, MacKersie A, Lane MS (2005) Derivation of a clinical guideline for the assessment of nonspecific abdominal pain: the Guideline for Abdominal Pain in the ED Setting (GAPEDS) Phase 1 Study. The American journal of emergency medicine 23 (6):709-717. doi:10.1016/j.ajem.2005.01.010

7. Netter FH (2014) Atlas of human anatomy. Sixth edition. edn. Saunders/Elsevier, Philadelphia, PA

8. Ecanow JS, Gore RM (2015) Evaluating Patients with Left Upper Quadrant Pain. Radiol Clin North Am 53 (6):1131-1157. doi:10.1016/j.rcl.2015.06.003 
9. Shuman WP, Ralls PW, Balfe DM, Bree RL, DiSantis DJ, Glick SN, Kidd R, Levine MS, Megibow AJ, Mezwa DG, Saini S, Greene FL, Laine LA, Lillemoe K (2000) Imaging evaluation of patients with acute abdominal pain and fever. American College of Radiology. ACR Appropriateness Criteria. Radiology 215 Suppl:209-212

10. Hammond NA, Nikolaidis P, Miller FH (2010) Left lower-quadrant pain: guidelines from the American College of Radiology appropriateness criteria. American family physician 82 (7):766770

11. Bree RL, Ralls PW, Balfe DM, DiSantis DJ, Glick SN, Levine MS, Megibow AJ, Saini S, Shuman WP, Greene FL, Laine LA, Lillemoe K (2000) Evaluation of patients with acute right upper quadrant pain. American College of Radiology. ACR Appropriateness Criteria. Radiology 215 Suppl:153-157

12. Ralls PW, Balfe DM, Bree RL, DiSantis DJ, Glick SN, Levine MS, Megibow AJ, Saini S, Shuman WP, Greene FL, Laine LA, Lillemoe K (2000) Evaluation of acute right lower quadrant pain. American College of Radiology. ACR Appropriateness Criteria. Radiology 215 Suppl:159166

13. Berkovich GY, Levine MS, Miller WT, Jr. (2000) CT findings in patients with esophagitis. AJR American journal of roentgenology 175 (5):1431-1434. doi:10.2214/ajr.175.5.1751431 